\title{
Dosimetry advantages of intraoperatively built custom-linked seeds compared with loose seeds in permanent prostate brachytherapy
}

\author{
Masahiro Inada, MD', Masaki Yokokawa, MD', Takafumi Minami, MD, PhD², Kiyoshi Nakamatsu, MD, PhD', \\ Yasumasa Nishimura, MD, $\mathrm{PhD}^{\mathrm{l}}$ \\ 'Department of Radiation Oncology, ${ }^{2}$ Department of Urology, Kindai University Faculty of Medicine, Osakasayama, Osaka, Japan
}

\begin{abstract}
Purpose: The aim of this study was to compare the implant quality between intraoperatively built custom-linked seeds (IBCL) and loose seeds (LS) retrospectively.

Material and methods: This study included 74 prostate cancer patients who were treated with permanent prostate brachytherapy (PPB) using IBCL $(n=37)$ or LS $(n=37)$ between July 2014 and June 2016. Dose-volume histogram (DVH) parameters, seed migration, and operation time were compared between the IBCL and LS groups. In addition to the standard target volume of the whole prostate gland, DVH parameters for prostate plus a $3 \mathrm{~mm}$ margin (CTV) were evaluated.

Results: In intraoperative planning, prostate $\mathrm{V}_{150}$ was lower $(54.8 \%$ vs. $59.6 \%, p=0.027)$, and CTV $\mathrm{V}_{100}(88.1 \%$ vs. $85.6 \%, p=0.019)$ and $\mathrm{D}_{90}(98.5 \%$ vs. $92.6 \%, p=0.0033)$ were higher in the IBCL group compared with in the LS group. In post-implant dosimetry, prostate $\mathrm{V}_{100}(96.9 \%$ vs. $95.2 \%, p=0.020)$, CTV V $100(85.6 \%$ vs. $81.7 \%, p=0.046)$, and CTV $\mathrm{D}_{90}(94.2 \%$ vs. $86.5 \%, p<0.001)$ were higher, and prostate $\mathrm{V}_{150}(57.1 \%$ vs. $64.5 \%, p=0.0051)$ and CTV $\mathrm{V}_{150}(31.5 \%$ vs. $35.7 \%, p=0.046)$ were lower in the IBCL group compared with in the LS group. Regarding DVH changes between intraoperative planning and post-implant dosimetry, the decrease in prostate $\mathrm{D}_{90}$ was significantly lower in the IBCL group than in the LS group $(-1.16 \%$ vs. $-4.17 \%, p<0.001)$. For the IBCL group, the operation time was slightly but significantly longer than that for the LS group ( 50.5 minutes vs. 43.7 minutes, $p=0.011$ ). However, the seed migration rate was significantly lower in the IBCL group than in the LS group ( $5 \%$ vs. $41 \%, p<0.001)$.

Conclusions: Intraoperatively built custom-linked is more advantageous than LS in terms of dosimetric parameters and migration.

J Contemp Brachytherapy 2017; 9, 5: 410-417 DOI: https://doi.org/10.5114/jcb.2017.70902
\end{abstract}

Key words: brachytherapy, intraoperatively built custom-linked seeds, iodine-125, prostate cancer, seeds.

\section{Purpose}

Permanent prostate brachytherapy (PPB) is a wellestablished method to treat localized prostate cancer [1,2, $3,4]$. Consequently, over the past decade, the number of prostate cancer patients who received PPB for initial local treatment has increased in Japan [5]. Several studies have demonstrated that a high $\mathrm{D}_{90}$ for the clinical target volume (CTV) improved biochemical relapse-free survival in prostate cancer patients treated with PPB $[1,2,4,6,7,8]$. However, high radiation doses to the urethra and rectum caused greater genitourinary and gastrointestinal toxicities $[9,10,11]$.

Several studies have reported that extraprostatic extension (EPE) occurs in 10-50\% of low-risk prostate cancer patients $[12,13,14,15,16]$. To ensure the potential sites for EPE are covered, defining the treatment margins is essen- tial [15]. Therefore, the European Society for Radiotherapy and Oncology (ESTRO) recommends that CTV corresponds to the whole prostate gland with a $3 \mathrm{~mm}$ margin [16]. Periphery seed placements are required to deliver the prescribed dose to the CTV; however, seeds implanted into peripheral area are accidentally replaced into extracapsular area, and easily migrate into distant area [17]. As seed migration results in poor dose distribution, seed movements should be minimized. Loose seeds (LS) and strand type seeds have been used in PPB. For the loose seeds method, seeds are placed into an extracapsular area; however, they can easily migrate to distant sites $[17,18]$. To mitigate this issue, strand type seeds were used for periprostatic seed loading $[19,20]$. As a type of stranded seed, intraoperatively built custom-linked seeds (IBCL) have been used in Japan since 2012. IBCL is a method that

Address for correspondence: Masahiro Inada, MD, Department of Radiation Oncology, Kindai University Received: 05.06.2017 Faculty of Medicine, Ohno-Higashi 377-2, Osakasayama, Osaka, Japan, phone: +81 72-366-0221, 
combines seeds and connectors into seed trains of variable length and seed-to-seed spacing [21]. IBCL has advantages of less seed migration and stability due to intraoperative linking $[22,23,24,25]$. This IBCL method may improve the dose volume histogram of the target and organs at risk (OAR) compared with conventional LS. In addition, IBCL was also advantageous in intraoperative customization compared with suture-embedded type strand seeds [26]. However, several studies have reported the advantages and disadvantages of IBCL [21,22,23,24,25]. Although IBCL reduces seed migration by linking seeds to each other, the nature of this feature also prolongs the operation time compared with LS. So far, three reports have demonstrated slight improvements in the dosimetric parameters for the prostate, urethra, and rectum by IBCL compared with LS [21,23,24]. Nevertheless, these reports only evaluated the dosimetric parameters of the whole prostate gland without evaluating those of CTV.

From July 2014, we began implementing IBCL in PPB. We hypothesized that IBCL improves the dosimetric parameters of CTV due to stable peripheral seed placement. The aim of this study was to compare the implant quality between IBCL and LS.

\section{Material and methods}

\section{Patient characteristics}

In June 2016, the retrospective analysis protocol was approved by the Investigational Review Board of the Faculty of Medicine of Kindai University. Between July 2014 and June 2016, all 74 prostate cancer patients who were treated with PPB using IBCL or LS in this period were subsequently enrolled in this study. Patient summary and treatment characteristics are shown in Table 1. Initial prostate cancer risk classifications were performed according to the National Comprehensive Cancer Network Guidelines [27]. During the aforementioned period, two patients were treated with PPB on the same day. One patient was treated with IBCL and the other was treated with LS. Two radiation oncologists decided the type of seeds to be used. In some cases, we selected IBCL for patients with very small prostates because we believed that IBCL would improve the post-implant dosimetry of the prostate.

\section{Brachytherapy technique}

To determine the number of seeds to be used, all patients underwent a transrectal-ultrasound examination (TRUS) 2-4 weeks prior to implantation. The prescribed brachytherapy dose was $144 \mathrm{~Gy}$ for the monotherapy, and 100 or 110 Gy for the combined therapy group. The combined therapy group further received supplemental external beam radiotherapy (sEBRT) with 40 or 45 Gy in 20 or 25 fractions. For combined therapy, intermediate-risk disease was treated with 100 Gy of brachytherapy, followed by $40 \mathrm{~Gy} / 20$ fractions of sEBRT, and high-risk disease was treated with $110 \mathrm{~Gy}$ of brachytherapy, followed by 45 Gy/25 fractions of sEBRT according to the National Comprehensive Cancer Network Guidelines. On the day of implantation, lumbar anesthesia was performed by the urologist. TRUS images in the axial plane were imported into the Variseed (Varian Medical Systems, Palo Alto, CA, USA) brachytherapy planning system at $2.5 \mathrm{~mm}$ slice spacing. The prostate, urethra, and rectal wall were contoured and reviewed by a single radiation oncologist. The CTV was defined as the prostate contour surrounded by a $3 \mathrm{~mm}$ margin that excluded the rectal wall. The initial

Table 1. Patient summary and treatment characteristics

\begin{tabular}{|c|c|c|c|}
\hline Factor & $\mathrm{IBCL}$ group $(n=37)$ & LS group $(n=37)$ & $p$-value \\
\hline Age (years) & Range, 54-80; Median, 68 & Range, 50-77; Median, 70 & 0.88 \\
\hline T stage & & & 0.83 \\
\hline $1 c / 2 a / 2 b / 2 c / 3 a$ & $13 / 15 / 3 / 3 / 3$ & $13 / 15 / 3 / 5 / 1$ & \\
\hline Gleason score & & & 0.33 \\
\hline $3+3 / 3+4 / 4+3 / \geq 8$ & $12 / 9 / 4 / 12$ & $15 / 14 / 2 / 6$ & \\
\hline PSA (ng/ml) & Range, 3.5-38; Median, 6.7 & Range, 3.4-17.6; Median, 6.0 & 0.53 \\
\hline Risk classification & & & 0.11 \\
\hline Low/Intermediate/High & $10 / 13 / 14$ & $12 / 19 / 6$ & \\
\hline Positive biopsy rate (\%) & Range, 6-100; Median, 20 & Range, 5-55; Median, 17 & 0.12 \\
\hline Neo-adjuvant hormonal therapy & & & 0.030 \\
\hline Yes/No & $18 / 19$ & $9 / 28$ & \\
\hline Prescribed BT dose & & & 0.99 \\
\hline 144 Gy fr monotherapy & 18 & 21 & \\
\hline 100 or 110 Gy for combined therapy & 19 & 16 & \\
\hline
\end{tabular}

$I B C L$ - intraoperatively built-custom link seeds, $L S$ - loose seeds, PSA - prostate specific antigen, BT-brachytherapy 
intraoperative plan was developed using the Nomogram Planning module (CR Bard, Covington, GA, USA). The seed number and location were modified manually from the initial plan as the modified uniform loading strategy [28]. In general, $70-80 \%$ of seeds were inserted in the peripheral prostate area and $20-30 \%$ of seeds were loaded to the internal area, and planned extracapsular placement was not performed in both the IBCL and LS groups. The loading technique, seed distribution, and dose-volume histogram (DVH) goal were not adjusted regardless of the type of seed or prescribed brachytherapy dose. Especially, planned extracapsular placement was not performed in both LS and IBCL groups. Based on the initial modifications, DVH and dose distribution were re-calculated using a real-time intraoperative dosimetry technique. The DVH goals in the intraoperative plan were as follows: $\mathrm{V}_{100}$ of prostate (the percentage of prostate volume receiving at least $100 \%$ of the prescribed dose) $>95 \%$; $\mathrm{D}_{90}$ of prostate (dose to $90 \%$ of prostate volume) between $115-135 \%$ of prescribed dose; $\mathrm{UD}_{10 \%}$ (dose to $10 \%$ of urethral volume) $<140 \%$ of prescribed dose; $\mathrm{RD}_{2 \mathrm{cc}}$ (dose to $2 \mathrm{cc}$ of rectal volume) $<100 \%$ of prescribed dose. The CTV dosimetry parameters were evaluated, although each CTV parameter was not made as an intraoperative planning DVH goal. After the initial plan was completed, 2 to 4 needles were inserted into the peripheral posterior sector to fix the prostate. Needle insertion was performed by a single urologist (TM). Peripheral needles and internal needles were inserted from the anterior sector and from the posterior sector under guidance from TRUS axial imaging. For careful rectal dose, needles near the rectum were inserted under the guidance of TRUS sagittal imaging. The final intraoperative planning dosimetry was fixed after re-calculation based on deposited seed location determined by TRUS images. Loose seeds (Oncoseed; GE Healthcare, Medi-Physics, Arlington Heights, IL, USA) were implanted using a Mick applicator (Mick Radio Nuclear Instruments, Mount Vernon, NY, USA). IBCL seeds (Brachysource; CR Bard, Covington, GA, USA) were assembled using a Quicklink device (CR Bard, Covington, GA, USA) prior to implantation. Zauls et al. [23] have described the detailed mechanisms of constructing IBCL, and we applied the same devices in our study. The operation time was obtained from the patient's exposure records. Operation time was defined as the time from the first seed implanted to the last seed implanted.

\section{Post-implant analysis}

Post-implant dosimetry was performed using computed tomography (CT) images taken 1 month after implantation. In these scans, $0.625 \mathrm{~mm}$ thick slice images were obtained. As urinary catheters were not employed in post-implant CT images, urethra contours were identified proportionally to the location on the planning ultrasound. A single radiation oncologist performed the post-implant dosimetry for all patients. The prostate, urethra, and rectal wall were contoured. To compare the DVH changes between TRUS-based intraoperative planning and post-implant analysis, the CTV was identified in the same way as in intraoperative planning. The rectal wall was contoured using the same slices that were used for the CTV contour.
In both intraoperative planning and post-implant dosimetry, the values for $\mathrm{V}_{100}, \mathrm{~V}_{150}$, and $\mathrm{D}_{90}$ of the prostate, and $\mathrm{CTV}$, urethral $\mathrm{D}_{10}$, and rectal $\mathrm{D}_{2 \mathrm{cc}}$ were calculated.

The incidence of seed migration was evaluated using chest-abdomen-pelvic plain X-ray films and CT exams obtained 1 day and 1 month after implantation. The number of migrated seeds was counted using X-ray, and detailed site of migration and distance from the prostate were evaluated using CT. Seed migration was defined as a seed distant from the prostate $(\geq 1.5 \mathrm{~cm})$. A seed that dropped into the base of the seminal vesicle $(<1.5 \mathrm{~cm}$ from the prostate) was not defined as a migrated seed.

\section{Statistical analysis}

The two-sample Welch's t-test for continuous data and $x^{2}$ test for categorical data were used to compare the baseline characteristics and the outcomes of interest between the IBCL and LS groups. One-way analysis of variance was used to compare the changes between intraoperative planning and post-implant dosimetry. Repeated measure analysis of variance was used to test the relationship between IBCL and LS on comparison of intra-operative planning, and post-implant dosimetry and prostate volume. Probability $(p)$ values of $<0.05$ were considered significant. Data processing and statistical analyses were carried out using Ekuseru-Toukei 2012 (Social Survey Research Information Co., Ltd, Tokyo, Japan).

\section{Results}

\section{Demographics}

Post-implant dosimetry analysis was performed for all 74 patients. There was a significant difference between the IBCL and LS groups in the number of patients undergoing neo-adjuvant hormonal therapy (Table 1). However, no significant differences were observed in the patients' age or risk classifications.

\section{Dosimetric parameters}

There was no significant difference between the two groups in prostate volume, implanted seed number, seed activity, or seed number per prostate volume (Table 2). On comparison of planned dosimetry, the $V_{150}$ of the prostate was lower $(54.8 \%$ vs. $59.6 \%, p=0.027)$, and $\mathrm{V}_{100}(88.1 \%$ vs. $85.6 \%, p=0.019)$ and $\mathrm{D}_{90}(98.5 \%$ vs. $92.6 \%$, $p=0.0033$ ) of CTV were higher in the IBCL group than in the LS group. No significant differences were observed in urethral or rectal dose. Table 3 shows the post-implant dosimetry. The prostate $\mathrm{V}_{100}(96.9 \%$ vs. $95.2 \%, p=0.020)$, CTV $V_{100}(85.6 \%$ vs. $81.7 \%, p=0.0012)$, and $\mathrm{D}_{90}(94.2 \%$ vs. $86.5 \%, p<0.001)$ were higher, and $\mathrm{V}_{150}$ of the prostate (57.1\% vs. $64.5 \%, p=0.020)$ and CTV $(31.5 \%$ vs. $35.7 \%$, $p=0.046)$ were lower in the IBCL group. Figure $1 \mathrm{de}-$ scribes the change in DVHs from intraoperative planning to post-implant dosimetry. The data revealed significant interactions between type of seed and DVH changes in prostate $\mathrm{D}_{90}(-1.16 \%$ vs. $-4.17 \%, p<0.001)$ and rectal dose $(-5.78 \%$ vs. $-0.36 \%, p=0.024)$. In the LS group, prostate volume significantly decreased compared with intra- 
Table 2. Intraoperative planning

\begin{tabular}{lccc} 
Factor & IBCL group $(n=37)$ & LS group $(n=37)$ & $p$-value \\
\hline Prostate volume $(\mathrm{ml})$ & $24.9(19.2-32.9)$ & $28.3(26.1-35.2)$ & 0.086 \\
\hline Implanted seed number & $62(50-79)$ & $70(60-75)$ & 0.24 \\
\hline $\begin{array}{l}\text { Seed activity (number of patients) } \\
11.0 \mathrm{MBq} / 13.1 \mathrm{MBq}\end{array}$ & $31 / 6$ & $32 / 5$ & 0.74 \\
\hline Seed number/Prostate volume $(\mathrm{ml})$ & $2.59(2.26-2.80)$ & $2.36(2.11-2.67)$ & 0.087 \\
\hline DVHs in US plan & & $96.9(1.88)$ & 0.13 \\
\hline Prostate $\mathrm{V}_{100}(\%)$ & $97.7(2.10)$ & $59.6(9.76)$ & 0.027 \\
\hline Prostate $\mathrm{V}_{150}(\%)$ & $54.8(8.08)$ & $119.6(8.20)$ & 0.55 \\
\hline Prostate $\mathrm{D}_{90}(\%$ of PD) & $120.9(9.93)$ & $85.6(4.72)$ & 0.019 \\
\hline $\mathrm{CTV}_{100}(\%)$ & $88.1(4.17)$ & $45.5(7.88)$ & 0.34 \\
\hline $\mathrm{CTV}_{150}(\%)$ & $43.9(6.63)$ & $92.6(8.00)$ & 0.0033 \\
\hline $\mathrm{CTV}_{90}(\%$ of PD) & $98.5(8.24)$ & $129.7(10.32)$ & 0.56 \\
\hline $\mathrm{UD}_{10}(\%$ of PD) & $131.1(9.15)$ & $64.5(5.39)$ & 0.17
\end{tabular}

Prostate volume, implanted seed number, and seed number/prostate volume are shown as medians (interquartile range: $25^{\text {th }}$ percentile - $75^{\text {th }}$ percentile). Dose volume histograms are shown as means (standard deviation)

$I B C L$ - intraoperatively built-custom link seeds, LS - loose seeds, US - ultrasound, PD - prescribed dose, $V_{100}$ - target volume receiving at least $100 \%$ of PD, $V_{150}$-target volume receiving at least $150 \%$ of $P D, D_{90}$ - percentage of $P D$ to $90 \%$ of target volume, $U D_{10}$ - percentage of $P D$ to $10 \%$ of urethral volume, $R D_{2 c c}-$ percentage of PD to 2 cc of rectal volume

Table 3. Post-implant dosimetry at 1 month

\begin{tabular}{lccc} 
Factor & IBCL group $(n=37)$ & LS group $(n=37)$ & $p$-value \\
\hline Prostate volume $(\mathrm{ml})$ & $24.8(22.5-31.0)$ & $26.7(24.7-32.9)$ & 0.27 \\
\hline Prostate $\mathrm{V}_{100}(\%)$ & $96.9(2.87)$ & $95.2(2.92)$ & 0.020 \\
\hline Prostate $\mathrm{V}_{150}(\%)$ & $57.1(11.27)$ & $64.5(10.55)$ & 0.0051 \\
\hline Prostate $\mathrm{D}_{90}(\%$ of PD) & $119.8(11.65)$ & $115.5(10.14)$ & 0.10 \\
\hline CTV $\mathrm{V}_{100}(\%)$ & $85.6(4.34)$ & $81.7(5.27)$ & 0.0012 \\
\hline CTV $\mathrm{V}_{150}(\%)$ & $31.5(8.14)$ & $35.7(7.68)$ & 0.046 \\
\hline CTV D $_{90}(\%$ of PD) & $94.2(9.34)$ & $86.5(8.52)$ & $<0.001$ \\
\hline $\mathrm{UD}_{10}(\%$ of PD) & $141.2(14.10)$ & $145.5(15.95)$ & 0.23 \\
\hline RD $_{2 c c}(\%$ of PD) & $61.0(10.18)$ & $64.1(11.15)$ & 0.23
\end{tabular}

Prostate volume is shown as median (interquartile range: $25^{\text {th }}$ percentile $-75^{\text {th }}$ percentile). Dose volume histograms are shown as means (standard deviation) $I B C L$ - intraoperatively built-custom link seeds, LS - loose seeds, US - ultrasound, PD - prescribed dose, $V_{100}-$ target volume receiving at least $100 \%$ of PD, $V_{150}$ - target volume receiving at least $150 \%$ of $P D, D_{90}$ - percentage of $P D$ to $90 \%$ of target volume, $U D_{10}$ - percentage of $P D$ to $10 \%$ of urethral volume, $R D_{2 c c}-$ percentage of PD to 2 cc of rectal volume

operative planning and post-implant dosimetry $(-1.56 \mathrm{ml}$, $p<0.001)$. A significant correlation between type of seed and volume change was observed $(-0.17 \mathrm{ml}$ for IBCL group vs. $-1.56 \mathrm{ml}$ for LS group, $p=0.011)$.

\section{Operation time and seed migration}

Table 4 describes the operation time and details of the site of seed migration. In the IBCL group, the mean operation time was slightly but significantly longer than in the LS group (50.5 minutes vs. 43.7 minutes, $p=0.011$ ). The percentage of patients with seed migration in the IBCL group was significantly lower than in the LS group (5\% vs. $41 \%, p<0.001)$. In addition, no seed migration other than into the seminal vesicle was observed in the IBCL group.

\section{Discussion}

This study demonstrated the dosimetric advantages of IBCL over LS for PPB. Several reports comparing DVH 

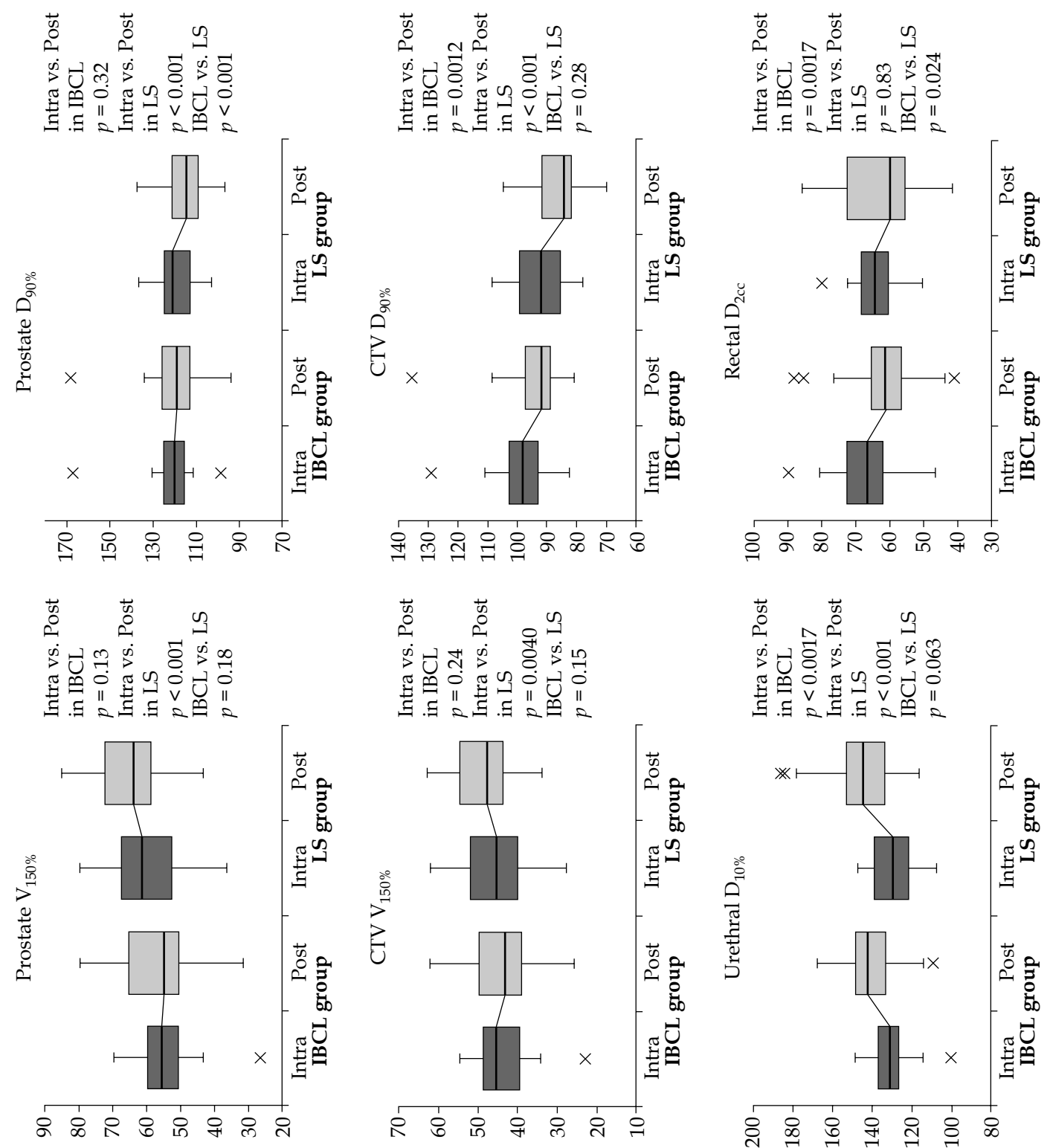

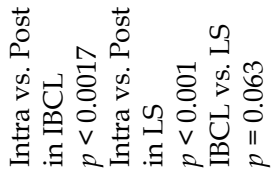
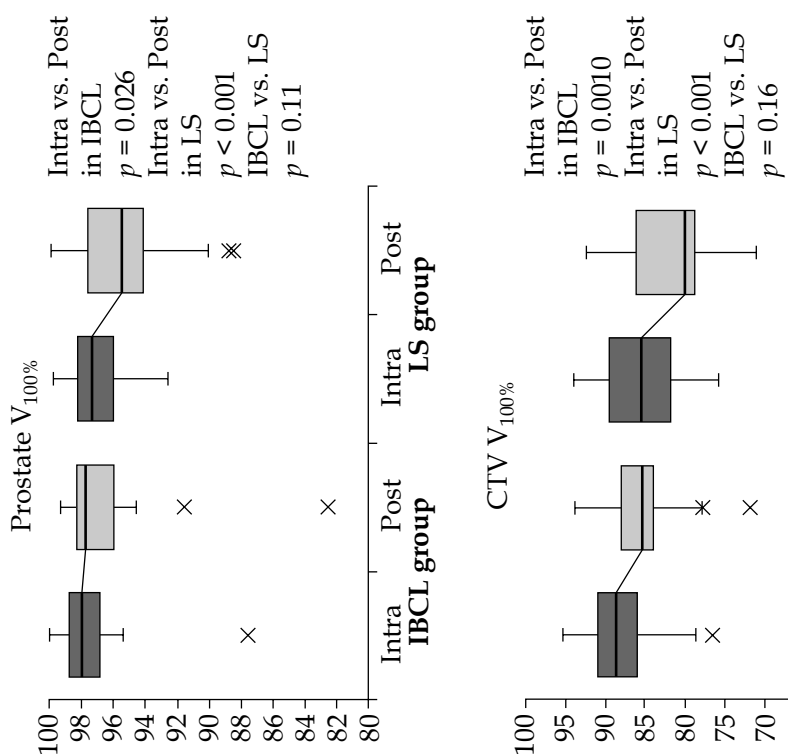

条务

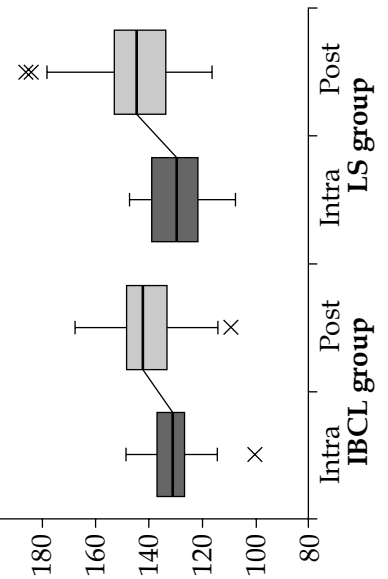

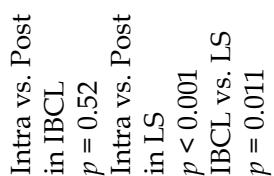
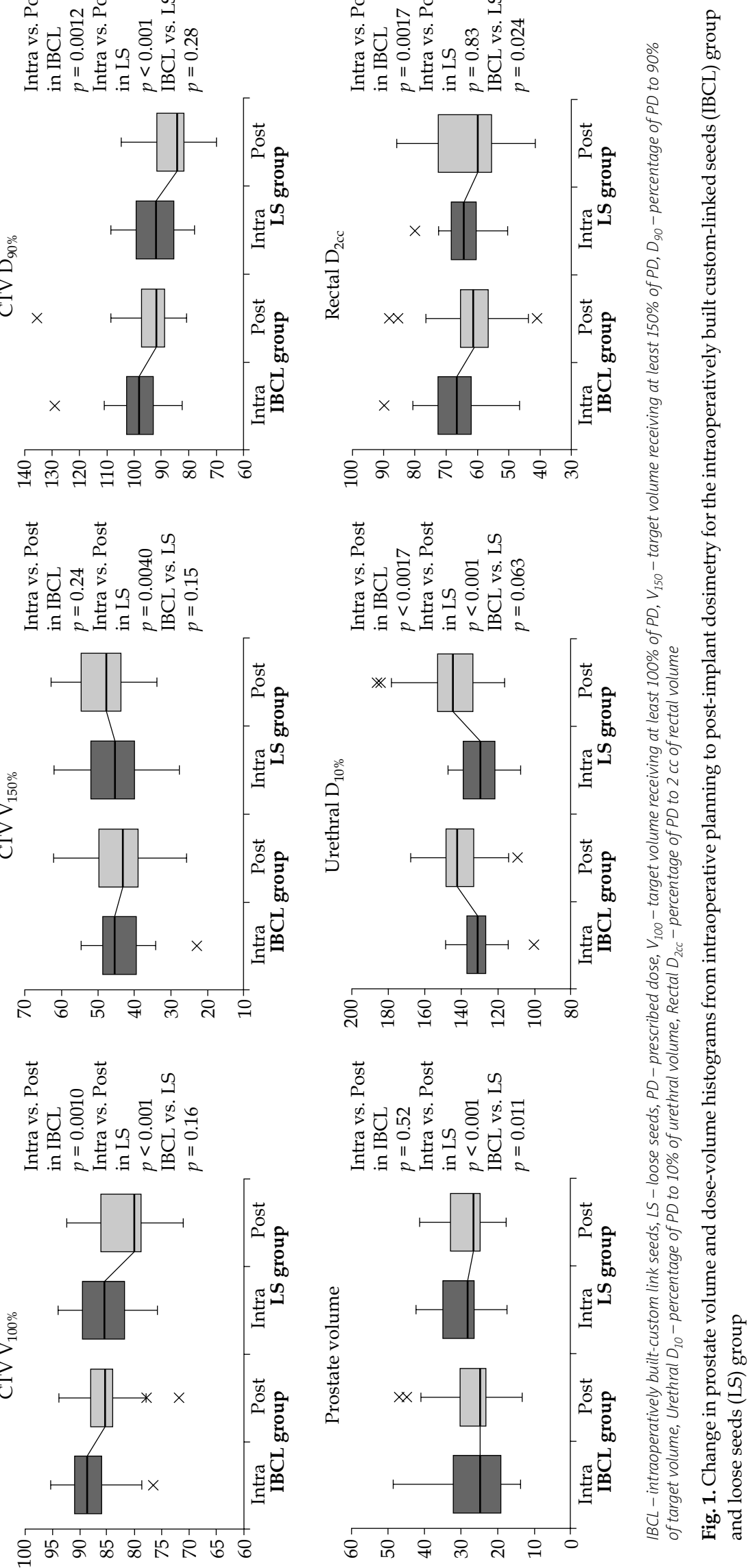
Table 4. Operation time and seeds migration

\begin{tabular}{lccc}
\hline Factor & IBCL group $(n=37)$ & LS group $(n=37)$ & $p$-value \\
\hline Operation time (min) & Mean, 50.5; SD 12.6 & Mean, 43.7; SD 9.0 & 0.011 \\
\hline Patient(s) with seeds migration & $2(5 \%)$ & $15(41 \%)$ & $<0.001$ \\
\hline Seminal vesicle & 2 & 6 & 8 \\
\hline Pelvic region & 0 & 2 &
\end{tabular}

$I B C L$ - intraoperatively built-custom link seeds, LS - loose seeds, SD - standard deviation

parameters between IBCL and LS found that prostate DVH did not differ between the two groups [22,23,24,25]. However, Jarusevicius et al. demonstrated that some DVH parameters were significantly different between IBCL and LS [21]. In contrast to the analysis methods used in the previous reports, this study compared DVH parameters that included the prostate with a $3 \mathrm{~mm}$ treatment margin (CTV). The CTV coverage was significantly higher in the IBCL group compared with in the LS group in both intraoperative planning and in post-implant dosimetry. It is thought that the periprostatic treatment margins are important for brachytherapy [7,8]. EPE is commonly seen in surgical specimens even at the early stages of the disease $[12,13,14,29]$. Sohayda et al. reported that most EPEs in low-risk prostate cancers were within $3.3 \mathrm{~mm}$ of the prostate [14]. Schwartz et al. also demonstrated that the risk of EPE over $6 \mathrm{~mm}$ in length was low for prostate cancers with favorable clinical parameters [29]. Therefore, delivering a greater radiation dose to the CTV is important. At the same time, the $\mathrm{V}_{150}$ of the prostate both in intraoperative planning and in post-implant dosimetry was significantly lower in the IBCL group compared with in the LS group. As higher $\mathrm{V}_{150}$ leads to greater urethral and bowel toxicities [30], reducing the $\mathrm{V}_{150}$ by IBCL may be beneficial for the patient's quality of life.

To deliver the prescribed dose to the target volume, radioactive seeds should be placed beneath and as close as possible to the prostate capsule. Though, LS not embedded in a vicryl structure were sometimes accidentally replaced into the extraprostatic area, and easily migrated to distant sites. In contrast, IBCL is more stable due to linkage and replacement into the extraprostatic area, and migrating to the distant sites is rare. This linkage allows the brachytherapist to place radioactive seeds in the beneath of the prostate capsule, and this feature improves dosimetry parameters of CTV. Although most EPE (within $3 \mathrm{~mm}$ ) can be treated by peripheral, but within the prostate, seed placement [31,32], the treatment margin was unstable due to several factors such as operator differences and post-implant volume change [31]. In addition, effects of source placement error may be large in peripheral dose [33]. An ongoing randomized phase 3 study testing BT monotherapy vs. BT with EBRT for intermediate-risk prostate cancer (RTOG-0232) demonstrated comparable biochemical control with BT monotherapy [34]. Therefore, implant quality for the treatment margin is required for brachytherapy. As such, stable seed location using
IBCL may be helpful in peripheral placement. In this analysis, we were able to apply a higher brachytherapy dose to CTV using IBCL without increasing seed migration. Although extraprostatic placement was not planned in this study, higher brachytherapy dose to CTV was achieved in the intraoperative plan. This may have occurred by seed replacement during implantation. As peripheral prostate gland dose is susceptible to seed movement, the extracapsular dose is also easily affected by seed movement [33]. Regarding $\mathrm{V}_{150}$, the high $\mathrm{V}_{150} \mathrm{~s}$ in the LS group may have been caused by seed overlap due to seed replacement during and after implantation. IBCL was more stable than LS due to seed linkage, reduced overlap, and decreased dose hot spots. Seeds stability may also have effects until 1 month after post-implant dosimetry. In this study, the decrease in prostate $D_{90}$ was lower in the IBCL group than in the LS group $(p<0.001)$. Limited reports were available comparing the oncological outcome between loose type and strand type of seeds. Hinnen et al. described an improved 5-year biochemical control for loose seeds compared with strand type seeds [35]. The author suspected that poorer post-implant prostate $\mathrm{D}_{90}$ values for the strand type group due to seed movement after implantation led to the poor biochemical control. In the trial, they used sutured-embedded seeds as strand seeds. Lower intraoperative customization ability should be observed with sutured-embedded type of strand seeds compared with IBCL [26]. In our study, the IBCL group achieved more stable prostate dosimetry compared with the LS group. It is known that a high biological effective dose in post-implant dosimetry is correlated with good biological control [36]. Thus, we believe that these seeds and dosimetric stability for IBCL may lead to improved oncological outcomes. However, in this study, prostate volume significantly decreased only in the LS group $(p<0.001)$ after implantation. This difference may have been caused by the significant difference in neo-adjuvant hormonal therapy use between the two groups (Table 1). Neo-adjuvant hormonal therapy is sometimes used to decrease prostate volume [37], although neo-adjuvant hormonal therapy is known to affect post-implant dosimetry. Ash et al. described that lower prostate $\mathrm{D}_{90}$ values were observed in patients treated with neo-adjuvant hormonal therapy due to prostate volume re-growth after seed implantation [38]. In this study, a significantly larger number of patients was treated with neo-adjuvant hormonal therapy in the IBCL group. Terminating hormonal 
therapy and the re-growth after implantation in the IBCL group caused significant prostate volume difference between the two groups. In the LS group, effects of prostate shrinkage after implant were suspected to be greater than in the IBCL group. However, the effect of volume reduction on dosimetry was expected to be a higher dose to the prostate [39], and the high prostate $\mathrm{D}_{90}$ decrease after implantation in the LS group may not have been affected by this volume reduction.

Several studies have reported the merits of IBCL over LS [21,22,23,24,25]. Jarusevicius et al. reported that IBCL improved prostate dose homogeneity, and decreased urethral and rectal dose for 160 Gy of the prescribed dose, whereas prostate $D_{90}$ in post-implant dosimetry was lower with IBCL compared with LS [21]. Katayama et al. compared IBCL and LS using sector analysis, and demonstrated that IBCL improved the anterior base prostate sector coverage for $144 \mathrm{~Gy}$ of the prescribed dose [25], whereas no significant difference was observed in whole prostate dosimetry. On the other hand, Ishiyama et al. found no dosimetric advantage with IBCL compared with LS for $145 \mathrm{~Gy}$ of the prescribed dose [22]. In contrast to this prior study, our results demonstrated that IBCL significantly improved the prostate $\mathrm{V}_{100}$ in post-implant dosimetry, and provided stable prostate $\mathrm{D}_{90}$ compared with LS. The difference in these results may be caused by inter-operator and/or inter-institute variability, including implanting strategy. Jarusevicius et al. hypothesized that seed movement during and after implantation in IBCL may reduce prostate $\mathrm{D}_{90}$ [21]. They reported that seed movement may occur by anchoring in the surrounding tissue [40]. In our study, as each seed was planned to be inserted inside the prostate, no seed movement inferior to the prostate apex was observed. Ishiyama et al. stated that they implanted several seeds outside the prostate gland only in the case of IBCL [22]; therefore, this different strategy may have contributed to the different results. Katayama et al. reported significantly higher intraoperative phase prostate $\mathrm{D}_{90}$ in the LS group, whereas this difference was not observed in post-implant dosimetry [25]. Although the dosimetric change was not tested in the study, IBCL may provide more stable dosimetry than LS. Our study had similar results with previous studies that reported less seeds migration and longer operation times.

There were several limitations to this study. First, this was a retrospective study, and there were several selection biases. In patient characteristics, the use of neo-adjuvant hormonal therapy was significantly higher in the IBCL group. This demographic difference may have affected the post-implant prostate volume and dosimetry. Second, as post-implant dosimetry analyses were performed using CT without a urethral catheter, there were uncertainties in the prostate and urethra delineations. However, as all post-implant dosimetry analyses were done by one radiation oncologist (MI), inter-observer variability was limited in this study. Third, we did not use Brachysource as loose seeds. Brachysource has gold-core in titanium capsules, different from Oncoseed. This gold-core marker improves fluoroscopic visibility; therefore, this difference in seeds components may affect intraoperative planning dosimetry.
Brachysource also causes minimal CT metal artifacts compared with Oncoseed. Post-implant dosimetry in Oncoseed patients may have more uncertainty in seeds identification and prostate contour compared with Brachysource. In addition, Brachysource has squared ends that aid in reducing seeds movement compared with Oncoseed, which has a rounded surface. To clarify the advantages of the intraoperatively built-custom system, comparison between IBCL and LS using the same Brachysource is warranted.

\section{Conclusions}

IBCL has several advantages over LS such as reduction in seeds migration. Furthermore, IBCL can help achieve high CTV coverage and prostate homogeneity in intraoperative planning, and prevent the decrease in prostate $D_{90}$ in 1 month post-implant dosimetry.

\section{Acknowledgements}

This work was supported by JSPS KAKENHI under grant numbers 16K10406.

\section{Disclosure}

Authors report no conflict of interest.

\section{References}

1. Morris WJ, Keyes M, Spadinger I et al. Population-based 10-year oncologic outcomes after low-dose-rate brachytherapy for low-risk and intermediate-risk prostate cancer. Cancer 2013; 119: 1537-1546.

2. Yorozu A, Kuroiwa N, Takahashi A et al. Permanent prostate brachytherapy with or without supplemental external beam radiotherapy as practiced in Japan: Outcomes of 1300 patients. Brachytherapy 2015; 14: 111-117.

3. Stokes SH. Comparison of biochemical disease-free survival of patients with localized carcinoma of the prostate undergoing radical prostatectomy, transperineal ultrasound-guided radioactive seed implantation, or definitive external beam irradiation. Int J Radiat Oncol Biol Phys 2000; 47: 129-136.

4. Kollmeier MA, Stock RG, Stone NN. Biochemical outcomes after prostate brachytherapy with 5-year minimal follow-up: importance of patient selection and implant quality. Int J Radiat Oncol Biol Phys 2003; 57: 645-653.

5. Onozawa M, Hinotsu S, Tsukamoto $\mathrm{T}$ et al. Recent trends in the initial therapy for newly diagnosed prostate cancer in Japan. Jpn J Clin Oncol 2014; 44: 969-981.

6. Stone NN, Potters L, Davis BJ et al. Multicenter analysis of effect of high biologic effective dose on biochemical failure and survival outcomes in patients with Gleason score 7-10 prostate cancer treated with permanent prostate brachytherapy. Int J Radiat Oncol Biol Phys 2009; 72: 341-346.

7. Choi S, Wallner KE, Merrick GS et al. Treatment margins predict biochemical outcomes after prostate brachytherapy. Cancer J 2004; 10: 175-180.

8. Crook J, Patil N, McLean M et al. Magnetic resonance imaging-defined treatment margins in iodine- 125 prostate brachytherapy. Int J Radiat Oncol Biol Phys 2010; 77: 1079-1084.

9. Zilli T, Taussky D, Le HP et al. Urethra-sparing, intraoperative, real-time planned, permanent-seed prostate brachytherapy: toxicity analysis. Int J Radiat Oncol Biol Phys 2011; 81: e377-383.

10. Ohashi T, Yozozu A, Saito $S$ et al. Urinary and Rectal Toxicity Profiles After Permanent Iodine-125 Implant Brachyther- 
apy in Japanese Men: Nationwide J-POPS Multi-institutional Prospective Cohort Study. Int J Radiat Oncol Biol Phys 2015; 93: 141-149.

11. Tanaka N, Asakawa I, Hasegawa M et al. Urethral toxicity after LDR brachytherapy: experience in Japan. Brachytherapy 2015; 14: 131-135.

12. Epestin JI, Partin AW, Sauvageot J et al. Prediction of progression following radical prostectomy. A multivariate analysis of 721 men with long-term follow-up. Am J Surg Pathol 1996; 20: 286-292.

13. Partin AW, Kattan MW, Subong EN et al. Combination of prostate-specific antigen, clinical stage, and Gleason score to predict pathological stage of localized prostate cancer. A multi-institutional update. JAMA 1997; 277: 1445-1451.

14. Sohayda C, Kupelian PA, Levin HS et al. Extent of extracapsular extension in localized prostate cancer. Urology 2000; 55: 382-386.

15. Merrick GS, Butler WM, Grimm P, et al. Permanent prostate brachytherapy extracapsular radiation dose distributions; analysis of multi-institutional database. J Contemp Brachytherapy 2013; 5: 117-121.

16. Salembier $C$, Lavagnini $P$, Nickers $P$ et al. Tumor and target volumes in permanent prostate brachytherapy: a supplement to the ESTRO/EAU/EORTC recommendations on prostate brachytherapy. Radiother Oncol 2007; 83: 3-10.

17. Eshleman JS, Davis BJ, Pisansky TM et al. Radioactive seed migration to the chest after interstitial prostate brachytherapy: extraprostatic seed placement correlates with migration. Int J Radiat Oncol Biol Phys 2004; 59: 419-425.

18. Reed DR, Wallner KE, Merrick GS et al. A prospective randomized comparison of stranded vs. loose $125 \mathrm{I}$ seeds for prostate brachytherapy. Brachytherapy 2007; 6: 129-134.

19. Merrick GS, Butler WM, Dorsey AT et al. Seed fixity in the prostate/periprostatic region following brachytherapy. Int J Radiat Oncol Biol Phys 2000; 46: 215-220.

20. Nobes JP, Khaksar SJ, Hawkins MA et al. Novel prostate brachytherapy technique: improved dosimetric and clinical outcome. Radiother Oncol 2008; 88: 121-126.

21. Jarusevicus L, Inciura A, Juzoaityte E et al. Comparison of implant quality between loose and intra-operatively linked iodine-125 seeds in prostate cancer brachytherapy. J Radiat Res 2012; 53: 439-446.

22. Ishiyama H, Satoh T, Kawakami S et al. A prospective quasirandomized comparison of intraoperatively built customlinked seeds versus loose seeds for prostate brachytherapy. Int J Radiat Oncol Biol Phys 2014; 90: 134-139.

23. Zalus AJ, Ashenafi MS, Onicescu G et al. Comparison of intraoperatively built custom linked seeds versus loose seed gun applicator technique using real-time intraoperative planning for permanent prostate brachytherapy. Int J Radiat Oncol Biol Phys 2011; 81: 1010-1016.

24. Ishiyama H, Satoh T, Yorozu A et al. Multi-institutional retrospective analysis of learning curves on dosimetry and operation time before and after introduction of intraoperatively built custom-linked seeds in prostate brachytherapy. J Radiat Res 2016; 57: 68-74.

25. Katayama N, Takemoto M, Takamoto A. Comparison of implant quality between intraoperatively built custom-linked seeds and loose seeds in permanent prostate brachytherapy using sector analysis. J Radiat Res 2016; 57: 393-399.

26. Guinot JL, Ricós JV, Tortajada MI et al. Comparison of permanent (125)I seeds implants with two different techniques in 500 cases of prostate cancer. J Contemp Brachytherapy 2015; 7: 258-264

27. NCCN Clinical Practice Guidelines in Oncology, Prostate Cancer, 2017.
28. Merrick GS, Butler WM. Modified uniform seed loading for prostate brachytherapy: rationale, design, and evaluation. Tech Urol 2000; 6: 78-84

29. Schwartz DJ, Sengupta S, Hillman DW et al. Prediction of radial distance of extraprostatic extension from pretherapy factors. Int J Radiat Oncol Biol Phys 2007; 69: 411-418.

30. Vordermark D, Noe M, Markert K et al. Prospective evaluation of quality of life after permanent prostate brachytherapy with I-125: importance of baseline symptoms and of prostate-V150. Radiother Oncol 2009; 91: 217-224.

31. Butzbach D, Waterman FM, Dicker AP. Can extraprostatic extension be treated by prostate brachytherapy? An analysis based on postimplant dosimetry. Int J Radiat Oncol Biol Phys 2001; 51: 1196-1199.

32. Patel AB, Waterman FM, Dicker AP. A detailed examination of the difference between planned and treated margins in 125I permanent prostate brachytherapy. Brachytherapy 2003; 2: 223-228.

33. Roberson PL, Narayana V, McShan DL et al. Source placement error for permanent implant of the prostate. Med Phys 1997; 24: 251-257.

34. BR Prestidge, K Winter, MG Sanda et al. Initial Report of NRG Oncology/RTOG 0232: A Phase 3 Study Comparing Combined External Beam Radiation and Transperineal Interstitial Permanent Brachytherapy With Brachytherapy Alone for Selected Patients With Intermediate-Risk Prostatic Carcinoma. Proceedings of the $58^{\text {th }}$ Annual Meetings of American Society for Radiation Oncology. 2016 September 25-28; Boston, America.

35. Hinnen KA, Moerland MA, Battermann JJ et al. Loose seeds versus stranded seeds in I-125 prostate brachytherapy: Differences in clinical outcome. Radiother Oncol 2010; 96: 30-33.

36. Stock RD, Stone NN, Tabert A et al. A dose-response study for I-125 prostate implants. Int J Radiat Oncol Biol Phys 1998; 41: 101-108.

37. Solhjem MC, Davis BJ, Pisansky TM et al. Prostate volume before and after permanent prostate brachytherapy in patients receiving neoadjuvant androgen suppression. Cancer J 2004; 10: 343-348.

38. Ash D, Al-Qaisieh B, Bottomley D et al. The impact of hormonal therapy on post-implant dosimetry and outcome following iodine-125 implant monotherapy for localised prostate cancer. Radiother Oncol 2005; 75: 303-306.

39. Crock J, McLean M, Yeung I et al. MRI-CT/fusion to assess post-brachytherapy prostate volume and the effects of prolonged edema on dosimetry following transperineal interstitial permanent prostate brachytherapy. Brachytherapy 2004; 3: 55-60.

40. Moerland MA, van Deursen MJ, Elias SG et al. Decline dose coverage between intraoperative planning and post implant dosimetry for I-125 permanent prostate brachytherapy: Comparison between loose and stranded seed implants. Radiother Oncol 2009; 91: 202-206. 